\title{
Erratum to: 'Characteristics of body composition and cardiometabolic risk of Japanese male heavyweight Judo athletes'
}

Hiroko Murata', Satomi Oshima ${ }^{2}$, Suguru Torii ${ }^{2}$, Motoko Taguchi ${ }^{2}$ and Mitsuru Higuchi ${ }^{*}$

Unfortunately, the original version of this article [1] contained an error. The caption for Fig. 1 was included incorrectly. The correct caption can now be found below.

Fig. 1 Comparison of prevalence in cardiometabolic risk in terms of blood biochemical parameters. Prevalence of cardiometabolic risk presents the percentage over the referenced normal range of respective parameters. The cut-off referenced normal range were as follows: AST $>40(\mathrm{U} / \mathrm{L})$, ALT $>45(\mathrm{U} / \mathrm{L}), \gamma-\mathrm{GTP} \geqq 80(\mathrm{U} / \mathrm{L})$, HDL-C $<40(\mathrm{mg} / \mathrm{dL}), \mathrm{LDL}-\mathrm{C}>140(\mathrm{mg} / \mathrm{dL}), \mathrm{TG} \geqq 150$ $(\mathrm{mg} / \mathrm{dL}), \mathrm{UA}>7.0(\mathrm{mg} / \mathrm{dL})$, HOMA-IR $\geqq 2.5$. "Significant differences compared with Nonheavyweight group, $p<0.05$.

\section{Author details}

${ }_{1}^{1}$ Graduate school of Sport Sciences, Waseda University, 112 Frontier Reseach Center 135-1 Horinouchi, Tokorozawa-shi, Saitama 359-1192, Japan. ${ }^{2}$ Faculty of Sport Sciences, Waseda University, 2-579-15 Mikajima, Tokorozawa-shi, Saitama 359-1192, Japan.

Received: 14 April 2016 Accepted: 14 April 2016

Published online: 19 April 2016

\section{Reference}

1. Murata H, Oshima S, Torii S, Taguchi M, Higuchi M. Characteristics of body composition and cardiometabolic risk of Japanese male heavyweight Judo athletes. J Physiol Anthropol. 2016;35:10.

\footnotetext{
* Correspondence: mhiguchi@waseda.jp

${ }^{2}$ Faculty of Sport Sciences, Waseda University, 2-579-15 Mikajima,

Tokorozawa-shi, Saitama 359-1192, Japan

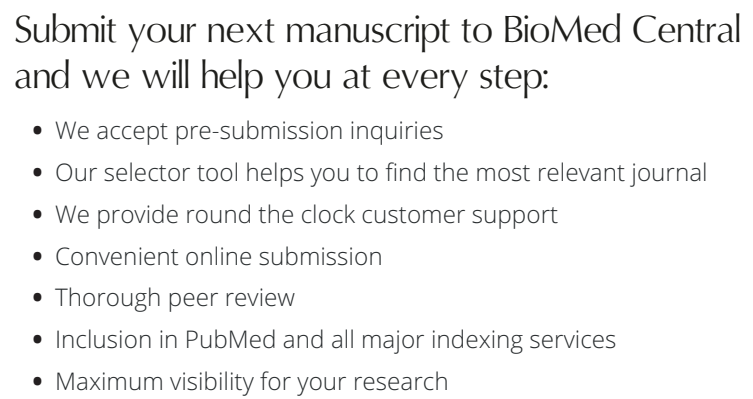

- We accept pre-submission inquiries

- Our selector tool helps you to find the most relevant journal

- We provide round the clock customer support

- Convenient online submission

- Thorough peer review

- Inclusion in PubMed and all major indexing services

- Maximum visibility for your research

Submit your manuscript at www.biomedcentral.com/submit 